\title{
ON IMPROVED RCM MODEL TO THEART EVALUATION FOR RADIATION SESOURCE
}

\author{
Ying Zhang $^{1 *}$ - Hong Wei Wang ${ }^{1,2}-$ Xiang Yu Fan ${ }^{1}-$ X.T. Guo ${ }^{1}$ \\ ${ }^{1}$ College of Aeronautics and Astronautics Engineering, Air Force Engineering University, Xi'an 710038, China \\ ${ }^{2}$ Electronic information college, North Western Polytechnical University, Xi'an 710072, China
}

\begin{tabular}{l} 
ARTICLE INFO \\
\hline Article history: \\
Received: 17.4 .2017$. \\
Received in revised form: 13.6 .2017$. \\
Accepted: 5.7.2017. \\
\hline Keywords: \\
Combined threat evaluation \\
Improved RCM \\
Coarse sorting \\
Radar operation Modes \\
Fine sorting \\
Radar chart \\
\hline DOI: http://doi.org/10.30765/er.40.1.03
\end{tabular}

\section{Introduction}

With the development of systematization and information warfare, the situation of battlefield has become more and more complex and changeable. As one of the vital technologies in modern electronic countermeasures [1], fast and accurate threat evaluation of hostile radiation resource is not only the precondition but also the foundation of accurate judgment of enemy's situation, and effective distribution of radar jamming resource. In addition to this, it is also very crucial for aircraft to accomplish operational mission and boost their own viability $[2,3]$.

In order to achieve threat evaluation of radiation resource in actual battlefield, many scholars have made some achievements in this field, for example,

\begin{abstract}
:
Focusing on the deficiency of intuition, real-time and complexity of threat evaluation of radiation resource, an algorithm based on improved radar chart method (RCM) is proposed in this paper. In the algorithm proposed, coarse sorting is integrated with fine sorting to obtain a more accurate and reliable result of threat evaluation. Coarse sorting is applied to sequence all the radiation resource roughly according to radar operation mode, and reduce the task priority of low-threat radiation resource. Then, on the basis of improved RCM, fine sorting is applied to sequence the radiation resource with same radar operation mode. Finally, obtain the results of threat evaluation which combined coarse sorting with fine sorting. Simulation analysis shows the correctness and effectiveness of this algorithm. Comparing with classical method of threat evaluation of radiation resource based on $R C M$, the algorithm proposed in this paper is more visual in image and can work quickly with lower complexity.
\end{abstract}

according to the fickle threat factors during beyondvisual-range air combat, Chen et al. [4] put forward a method based on improved glowworm swarm optimization (IGSO) algorithm and BP neural network. In the condition of no prior information, Wang et al. [5] proposed an evaluation algorithm which combined rough set (RS) and technique for order preference by similarity to solution (TOPSIS). In [6], Particle Swarm Optimization (PSO) is utilized to optimize penalty parameter $\boldsymbol{c}$ and core function $\boldsymbol{g}$ in support vector machine (SVM), therefore a new target threat assessment model based on PSO-SVM has been established. All the above methods can achieve threat evaluation of radiation resource in some situations, however, there are some limitations in practical application. Such as

\footnotetext{
${ }^{*}$ Corresponding author. Tel.: 13335386542

E-mail address: zhangying19930504@163.com
} 
the complex computation, and the unintuitive evaluation results.

On the basis of present studies and aiming at the limitation of above methods, an algorithm of threat evaluation of radiation resource is proposed in this article, which utilizes radar operation mode to accomplish coarse sorting and improved radar chart to achieve fine sorting. First of all, sequence all the radiation resource's threat level roughly according to radar operation mode, next, reduce the task priority of low-threat radiation resource. Secondly, on the basis of improved RCM, sequence the radiation resources with same radar operation mode nicely. Finally, combine the result of coarse sorting with fine sorting to obtain the eventual results of threat evaluation of radiation resource.

\section{The algorithm of improved RCM}

As a multivariate and graphical analysis method, radar chart utilizes graph form to reflect the quantitative relation of indexes, and combines the mathematical treatment of image features. Therefore, radar chart is capable of reflecting the comprehensive strength of evaluation targets, and the result is vivid and straightforward [7]. During the period of comprehensive quantitative evaluation, the angle of each index axis is divided equally in traditional radar chart, as a result, it can't reflect the influence of index's weight [8]. Ordinary normalization method is employed to quantify the index in traditional radar chart, and the rationality of it is quite poor. In addition to this, radar chart is very complicate and because of the enormous amount of radiation resources and indexes, real-time of radar chart is poor. Focusing on the problems above, a new algorithm based on improved RCM is proposed in this article. According to the combination weight of index, determine the size of sector domain of different index. Meanwhile, determine the normalized evaluation value of index utilizing intuitionistic fuzzy sets (IFS). The specific steps of improved RCM are as follows:

Step 1: The identification of target set $\boldsymbol{U}=\left\{x_{1}, x_{2}, \ldots\right.$, $\left.x_{\mathrm{n}}\right\}$ and index set $\boldsymbol{A}=\left\{a_{1}, a_{2}, \ldots, a_{\mathrm{m}}\right\}$.

Step 2: The identification of combination weight $c \omega_{j}(j=1,2, \ldots \mathrm{m})$ of index. In order to obtain a more scientific and reasonable weight of index, a combination weight which combined subjective weight $s \omega_{j}$ with objective weight $p \omega_{\mathrm{j}}$ is proposed in this section.
First of all, according to equation (1), use intuitionistic fuzzy entropy ${ }^{[9]}$ to compute the objective weight $p \omega_{\mathrm{j}}$.

$$
p \omega_{j}=\frac{1-H_{j}}{\sum_{j=1}^{m}\left(1-H_{j}\right)}, j=1,2, \ldots, m
$$

Where, $H_{j}$ represents the intuitionistic fuzzy entropy and is computed by equation (2).

$$
H_{j}=\frac{1}{n} \sum_{i=1}^{n} \frac{1-\left|u\left(x_{i j}\right)-v\left(x_{i j}\right)\right|+\pi\left(x_{i j}\right)}{1+\left|u\left(x_{i j}\right)-v\left(x_{i j}\right)\right|++\pi\left(x_{i j}\right)}
$$

Where, $u\left(x_{i j}\right)(i=1,2, \ldots n ; j=1,2, \ldots m)$ is membership function which represents affirmative information of each index, and is computed by the known formula of membership degree in general. $v\left(x_{i j}\right)$ is nonmembership function which represents negative information of each index, and is computed by equation (3).

$$
v\left(x_{i j}\right)=1-u\left(x_{i j}\right)-\pi\left(x_{i j}\right)
$$

where, $\pi\left(x_{i j}\right)$ is intuitionistic fuzzy function which represents hesitancy degree of each index, and the calculation formula is given by different types of index. The specific solving process can be referred in literature [10].

Secondly, utilize group analytic hierarchy process (group-AHP) ${ }^{[11]}$ to compute the subjective weight $\mathrm{s} \omega_{\mathrm{j}}$. Construct the judgment matrix $Q_{\mathrm{k}}$ according to equation (4)

$$
Q_{k}=\left(q_{k}^{i j}\right) n \times m
$$

Where, there are $\boldsymbol{g}$ experts in total, and $\boldsymbol{Q}_{\boldsymbol{k}}$ is the judgment matrix of $\boldsymbol{k}$-th expert. If $\boldsymbol{Q}_{\boldsymbol{k}}$ can meet the conditions of conformance check, compute the subjective weight according to equation (5), otherwise adjust $\boldsymbol{Q}_{\boldsymbol{k}}$, [12] Refs.

$$
s \omega_{j}=\frac{\left[\prod_{j=1}^{m} \prod_{k=1}^{g} q_{j k}^{\varepsilon_{k}}\right]^{\frac{1}{m}}}{\sum_{i=1}^{n}\left[\prod_{j=1}^{m} \prod_{k=1}^{g} q_{j k}^{\varepsilon_{k}}\right]^{\frac{1}{m}}}
$$


Where, $\boldsymbol{\varepsilon}_{\boldsymbol{k}}$ represents the weight of experts and can be obtained through experience.

Finally, compute the combination weight which combined subjective weight $s \omega_{j}$ with objective weight $p \omega_{\mathrm{j}}$.

$$
c \omega_{j}=a \times s \omega_{j}+b \times p \omega_{j}, a, b \geq 0 ; a+b=1
$$

Where, $\boldsymbol{a}$ respects the coefficient of subjective weight, $\boldsymbol{b}$ respects the coefficient of objective weight, and they are gave by actual situation.

Step 3: Distribute the size of sector domain of each index according to the combination weight of index. Record the $\boldsymbol{j}$ th index's angle of sector domain in radar chart is $\theta_{j}=360 \omega_{j}$.

Step 4: The identification of index axis. Draw a unit circle, draw a ray ' $\mathbf{O A}$ ' from the unit circle's center ' $\mathbf{O}$ ', and ' $\mathbf{O A}$ ' cuts the unit circle at point ' $\mathbf{A}$ '. According to the angle of $\boldsymbol{m}$ indexes, draw the rest of $\boldsymbol{m}$ - 1 rays write for ' $\mathbf{O B}$ ' ' ' $\mathbf{O C}$ '..., then draw the angle bisectors of sector 'AOB', 'BOC'..., and cut the unit circle at point ' $\mathrm{P}_{1}$ ', ' $\mathrm{P}_{2}$ ', .. ' $\mathrm{P}_{\mathrm{m}}$ ', finally, regard ' $\mathrm{OP}_{1}$ ', .., ' $\mathrm{OP}_{\mathrm{m}}$ ' as the index axis.

Step 5: Draw radar chart. According to the evaluation value $r_{j}(j=1,2, \ldots \mathrm{m})$ of index, sign the corresponding points of index axis in radar chart and cut index axis at point 'A', 'B', 'C', 'D', .... Joint all the points and obtain the radar chart as shown in Fig. 1.

Where, the evaluation value of index $r\left(x_{i j}\right)$ is computed by equation (7), which referred to literature [13]. According to IFS, in order to obtain a more objective and accurate radar chart, we consider the influence of subjection function, non- membership function and hesitancy degree together as a whole.

$$
\begin{aligned}
& r\left(x_{i j}\right)= \\
& u\left(x_{i j}\right)+\left[1-u\left(x_{i j}\right)-v\left(x_{i j}\right)\right] \frac{u\left(x_{i j}\right)}{u\left(x_{i j}\right)+v\left(x_{i j}\right)}
\end{aligned}
$$

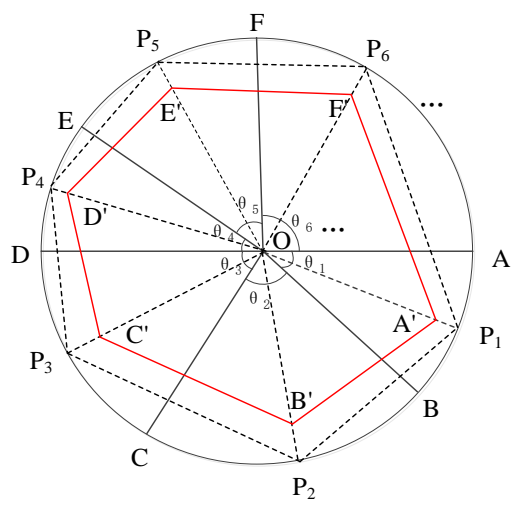

Figure 1. Improved radar chart

Step 6: Compute threat evaluation value ' $K$ '. Joint point ' $\mathrm{P}_{1}$ ', ' $\mathrm{P}_{2}$ ', ...' $\mathrm{P}_{\mathrm{m}}$ ' in turn in Fig. 1 to get $\mathrm{a}$ polygon ' $\mathrm{P}_{1} \mathrm{P}_{2} \ldots \mathrm{P}_{m}$ ' which is regarded as standard polygon. The size of polygon ' $\mathrm{P}_{1} \mathrm{P}_{2} \ldots \mathrm{P}_{m}$ ' is ' $\mathrm{S}$ ' and the perimeter is ' $C$ '. Joint point ' $A$ ', ' $B$ ', ' $C$ '... in turn in Fig. 1 and get a polygon $A^{\prime} B^{\prime} C^{\prime}$ '..., the size of polygon ' $A$ ' $B$ ' $C$ '. ... is ' $S$ ' and the perimeter is ' $C$ '. The computation formula of threat evaluation value ' $K$ ' is shown as follows:

$$
\begin{gathered}
S=\frac{1}{2}\left[\sum_{j=1}^{m} \sin \left(\frac{\theta_{i}+\theta_{i+1}}{2}\right)+\sin \left(\frac{\theta_{1}+\theta_{m}}{2}\right)\right] \\
L=\sum_{j=1}^{m} \sqrt{2-2 \cos \left(\frac{\theta_{i}+\theta_{i+1}}{2}\right)}+\sqrt{2-2 \cos \left(\frac{\theta_{1}+\theta_{m}}{2}\right)} \\
S^{\prime}=\frac{1}{2}\left[\sum_{j=1}^{m} r_{j} r_{j+1} \sin \left(\frac{\theta_{i}+\theta_{i+1}}{2}\right)+r_{1} r_{m} \sin \left(\frac{\theta_{1}+\theta_{m}}{2}\right)\right] \\
L^{\prime}=\sum_{j=1}^{m} \sqrt{r_{j}^{2}+r_{j+1}^{2}-2 \cos \left(\frac{\theta_{i}+\theta_{i+1}}{2}\right)}+\sqrt{r_{1}^{2}+r_{m}^{2}-2 \cos \left(\frac{\theta_{1}+\theta_{m}}{2}\right)} \\
k=f\left(\beta_{1}, \beta_{2}\right)=\sqrt{\beta_{1} \bullet \beta_{2}}
\end{gathered}
$$


Where: $\beta_{1}=S^{\prime} / S, \beta_{2}=1+\left|S-S^{\prime}\right| / S$

Step 7: Sort the threat degree of radiation resource according to threat evaluation value ' $\mathrm{K}$ '.

\section{Combined threat evaluation of radiation resource method based on improved RCM}

On the basis of improved RCM, an algorithm of combined threat evaluation of radiation resource is proposed in this article, it based on the coarse sorting which utilizes radar operation mode and the fine sorting which utilizes improved RCM. Firstly, sequence all the radiation resources roughly according to radar operation mode. Then, on the basis of improved RCM, sequence the radiation resource with same radar operation mode nicely. The coarse sorting result based on radar operation mode is regarded as initial result, and it has an advantage of timeliness. In addition, coarse sorting is integrated with fine recruit, so that we can eventually obtain a more accurate and reasonable result of threat evaluation of radiation resource.

\subsection{The index system of threat evaluation of radiation resource}

The traditional evaluation index system puts particular emphasis on the parameters of emitter signal, however, in the condition of actual complicated battlefield, just take signal parameters into account is nowhere near enough. Therefore, this article put forward an index system of threat evaluation which overall considers platform index and radar index, the specific index system is shown in Fig. 2.

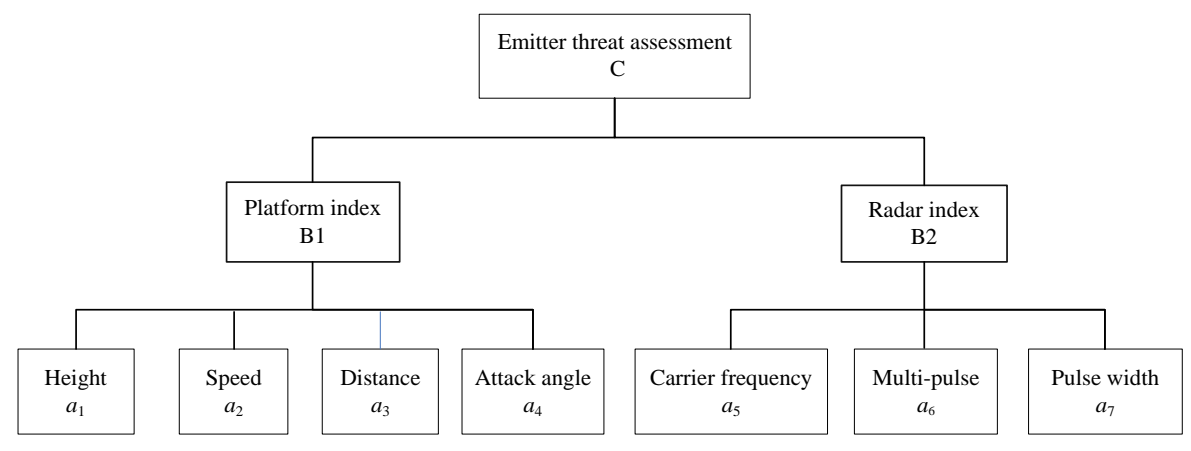

Figure 2. The index system of threat evaluation of radiation resource

\subsection{The process of combined threat evaluation of radiation resource based on improved $\mathrm{RCM}$}

The flow chart of combined threat evaluation of radiation resource based on improved RCM is shown as Fig. 3.

As shown in the Fig. 3, first of all, radar operation mode is used to sequence all the radiation resources roughly and quickly. Secondly, on the basis of improved RCM, sequence the radiation resources with same radar operation mode. Finally, combine the result of coarse sorting with fine sorting to obtain the eventual threat evaluation results. The specific steps are shown as follows:

Step 1: Construct the decision environment. Identify the target set $\boldsymbol{U}=\left\{x_{1}, x_{2}, \ldots, x_{n}\right\}$ and index set $\boldsymbol{A}=$ $\left\{a_{1}, a_{2}, \ldots, a_{7}\right\}$.

Step 2: Refer to literature [14], compute the radar operation mode of target set $\boldsymbol{U}$ according to radar index $a_{5}-a_{7}$. First of all, pre-divide the radar operation modes according to intra-pulse modulation and the range of PRF, after that, determine whether the operation mode belongs to VS (Velocity Search) or STT (Single Target Track). Secondly, according to equation (13) and (14), compute the reliability function of platform $\boldsymbol{j}$ to target mode $\boldsymbol{F}_{l}(l=1,2,3,4)$ and the reliability function for the uncertainty of the sensor $\boldsymbol{p}$ :

$$
\begin{aligned}
& \frac{m_{p}\left(F_{l}\right)=}{\sum_{l=1}^{N_{F}} C_{p}\left(F_{l}\right)+N\left(1-R_{p}\right)\left(1-W_{p} \alpha_{p} \beta_{p}\right)} \\
& m_{p}(\sigma)=1-\sum_{l=1}^{N_{F}} m_{p}\left(F_{l}\right)
\end{aligned}
$$

Where, $C_{P}\left(F_{l}\right)$ is the correlation coefficients of target mode $F_{l}$ with platform $\boldsymbol{p} . \boldsymbol{N}_{p}$ is the number of 
operation modes, $\boldsymbol{N}$ is the number of platforms, $\boldsymbol{W}_{p}$ is environment weighted coefficients of platform $\boldsymbol{p}$.

Thirdly, utilize DS evidence theory to achieve multi-periods data fusion under single platform and multi-sensors information fusion of $m_{p}\left(F_{l}\right)$ and $m_{p}(\sigma)$.

Finally, compare the fusion result with the threshold $\boldsymbol{\alpha}$ and $\boldsymbol{\beta}$ which respect DS classification decision rule, and then distinguish the operation modes TWS (Track While Scan) and RWS (Range While Search).

Step 3: Sequence all the radiation resources $\left(x_{1}-x_{\mathrm{n}}\right)$ roughly according to radar operation mode. There are four kinds of radar operation mode: Class 1 : STT > Class 2: TWS > Class 3: RWS > Class 4: VS. Step 4: When the radar operation mode are vary from emitter to emitter, output the result of threat evaluation of radiation resource according to Step 3 in Section 3.2. On condition that there are some emitters with same radar operation mode, then, sequence these emitters according to the Steps below.

Step 5: The identification of index's combination weight. According to Step 2 in Section 2, compute the combination weight of emitters with same radar operation mode, and the result is remarked as $\omega_{j}(j=1,2, \ldots 4)$. Where, $\omega_{j}$ represents the weight of platform index $\left(a_{1}-a_{4}\right)$.

Step 6: Draw radar chart. According to Step 3-Step 5 in Section 2, draw the radar chart of emitters with same operation mode.

Step 7: Compute threat evaluation value ' $\mathrm{K}$ '. According to Step 6 in Section 2, compute threat evaluation value ' $K$ ' of emitters with same operation mode. Then, obtain the final threat evaluation results which combined coarse sorting with fine sorting

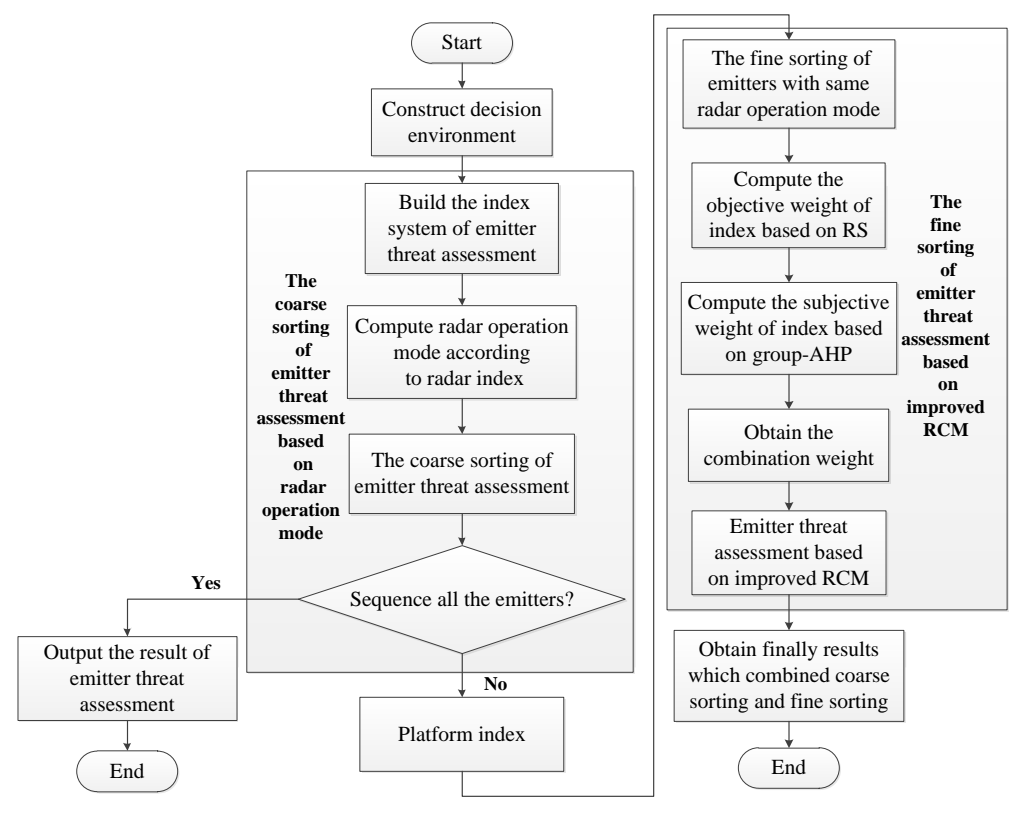

Figure 3. The flow chart of combined threat evaluation of radiation resource based on improved RCM

\section{Simulation and verification}

The simulation data refers to literature [7], target set $\boldsymbol{U}: x_{1}-\chi_{6}$; index set $\boldsymbol{A}: a_{1}-a_{7}$, where, $a_{1}$ : height $(\mathrm{km})$, $a_{2}$ : speed (Ma), $a_{3}$ : distance $(\mathrm{km}), a_{4}$ : attack angle $\left({ }^{\circ}\right), a_{5}$ : carrier frequency $(\mathrm{GHz}), a_{6}$ : multi-pulse $(\mathrm{kHz}), a_{7}$ : pulse width $(\mu \mathrm{s})$. The information of emitters at $\boldsymbol{t}$ o'clock are shown as Table 1 .
Table 1. The information of emitter at to'clock

\begin{tabular}{|c|c|c|c|c|c|c|c|}
\hline Emitter & $a_{1}$ & $a_{2}$ & $a_{3}$ & $a_{4}$ & $a_{5}$ & $a_{6}$ & $a_{7}$ \\
\hline$x_{1}$ & 6.8 & 1.8 & 72 & 14 & 32 & 102 & 0.3 \\
\hline$x_{2}$ & 7.8 & 0.9 & 190 & 17 & 14 & 32 & 4.6 \\
\hline$x_{3}$ & 6.2 & 1.3 & 120 & 13 & 21 & 47 & 1.2 \\
\hline$x_{4}$ & 5.4 & 1.4 & 113 & 12 & 19 & 51 & 2.6 \\
\hline$x_{5}$ & 8.4 & 0.4 & 213 & 20 & 4.3 & 5.6 & 5.9 \\
\hline 6 & 4.7 & 1.6 & 104 & 8 & 16 & 19 & 1.7 \\
\hline
\end{tabular}


The specific steps of the combined threat evaluation of radiation resource based on improved RCM are as follows:

1) Refer to the method in literature [14], according to Step 2 in Section 2, compute the radar operation mode of emitters $x_{1}-x_{6}$ based on data information $a_{5}$ $a_{7}$ in Table 1 . The result is : $x_{1}$ : STT, $x_{2}$ : RWS, $x_{3}$ : TWS, $x_{4}$ : TWS, $x_{5}$ : VS, $x_{6}$ : TWS.

2) The result of emitters' coarse sorting according radar operation mode shows that: $x_{1}>\left(x_{3}, x_{4} 、 x_{6}\right)>$ $x_{2}>x_{5}$. From the result available, emitter $x_{1}$ has the highest threat level of all emitters, and it is regarded as the first level. Emitter $x_{3} 、 x_{4} 、 x_{6}$ have same radar operation mode and they are regarded as the second level. Emitter $x_{2}$ is regarded as the third level and emitter $x_{5}$ is regarded as the fourth level.

3) According to Step 2 in Section 2, compute the index $\left(a_{1}-a_{4}\right)$ 's combination weight of emitter $x_{3} 、 x_{4} 、 x_{6}, \omega=(0.12,0.35,0.39,0.14)$. Then compute the angle of index $a_{1}-a_{4}$ in radar chart according to Step3 in Section $2, \theta=\left(43.2^{\circ}, 126^{\circ}\right.$, $\left.140.4^{\circ}, 50.4^{\circ}\right)$.

4) According to Step 5 in Section 2, compute the evaluation value of index $\left(a_{1}-a_{4}\right)$ of emitter $x_{3} 、 x_{4} 、 x_{6}, \mathrm{r}\left(x_{3}\right)=(0.47,0.77,0.87,0.48)$, $\mathrm{r}\left(x_{4}\right)=(0.51,0.79,0.88,0.49), \mathrm{r}\left(x_{6}\right)=(0.52,0.83$, $0.91,0.53)$. Then, draw the radar chart of emitter $x_{3} 、 x_{4}, x_{6}$ according to the value of ' $\boldsymbol{\theta}$ ' and ' $\mathbf{r}$ '.

5) Integrate Fig. 4、Fig. 5 and Fig. 6 to get the threat degree comparison of emitter $x_{3} 、 x_{4} 、 x_{6}$, the result is shown as Fig. 7.

6) Compute threat the evaluation value ' $\mathbf{K}$ ' of emitter $x_{3} 、 x_{4} 、 x_{6}$ according to Step 6 in Section 2:

$$
\begin{aligned}
& \mathrm{K}\left(x_{3}\right)=0.77 \\
& \mathrm{~K}\left(x_{4}\right)=0.81 \\
& \mathrm{~K}\left(x_{6}\right)=0.85
\end{aligned}
$$

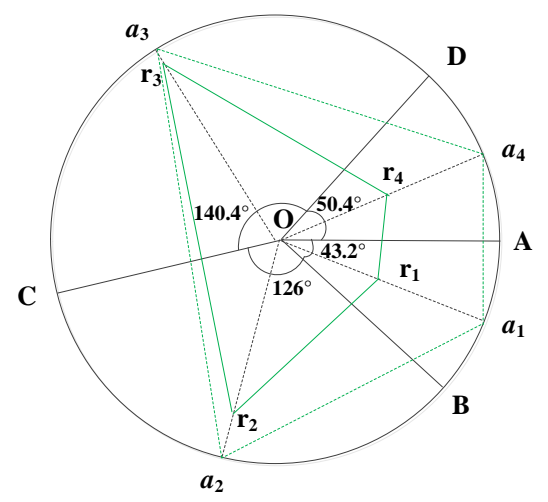

Figure 4. The radar chart of $x_{4}$

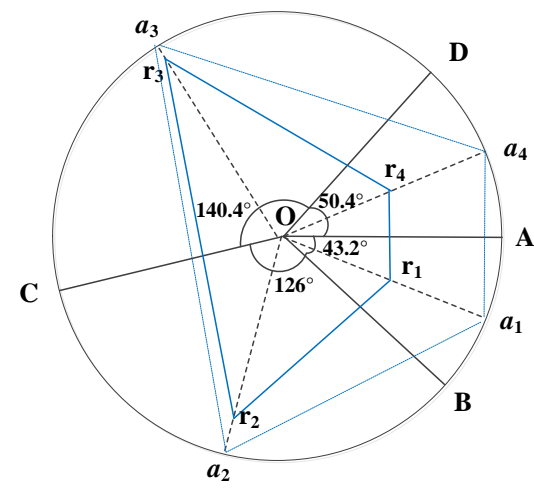

Figure 5. The radar chart of $x_{5}$

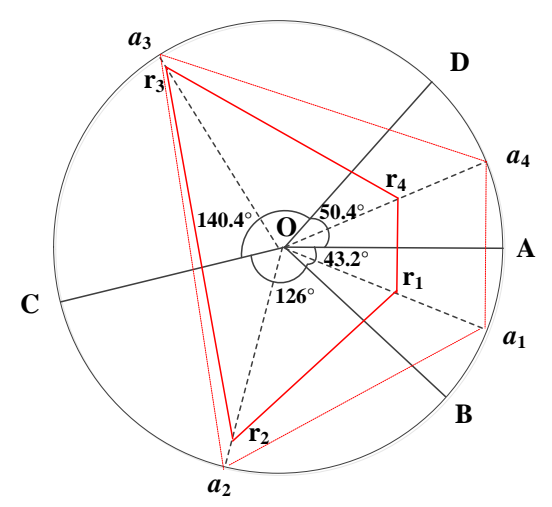

Figure 6. The radar chart of $x_{6}$

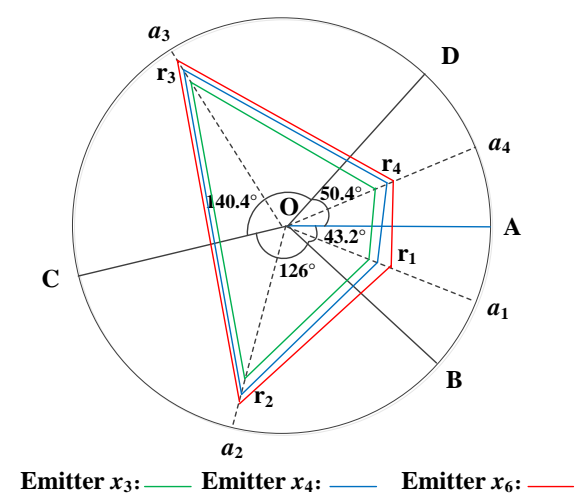

Figure 7. The contrast radar chart of emitter

$$
x_{3}, x_{4}, x_{6}
$$

According to the value of ' $\mathbf{K}$ ', sequence the threat level of emitter $x_{3} 、 x_{4} 、 x_{6}$. The result is: $x_{6}>x_{4}>x_{3}$. Then, combine the result with coarse sorting and obtain the final threat evaluation of emitters $x_{1}-x_{6}$ : $x_{1}>x_{6}>x_{4}>x_{3}>x_{2}>x_{5}$.

Through the analysis of Table 1 , it is obvious that of all the emitters $\left(x_{1}-x_{6}\right)$, emitter $x_{1}$ has the maximum 
speed, carrier frequency and multi-pulse, the minimum distance and pulse width, in the meantime, the radar operation mode of emitter $x_{1}$ is STT, in conclusion, the threat degree of $x_{1}$ is the greatest of all the emitters. Emitter $x_{5}$ has the maximum height 、distance、 attack angle and pulse width, the minimum speed carrier frequency and multi-pulse, in addition to this, the radar operation mode of emitter $x_{5}$ is VS, therefore, emitter $x_{5}$ has the minimal threat degree of all the emitters. The index information of emitter $x_{2}$ is very close to $x_{5}$, furthermore, the radar operation mode of $x_{2}$ is RWS and $x_{5}$ is VS, hence, the threat degree of $x_{2}$ is bigger than $x_{5}$. In the rest of emitters, the carrier frequency multi-pulse and pulse width of emitter $x_{3} 、 x_{4}$ and $x_{6}$ are quite close, besides this, the radar operation mode of emitter $x_{3} 、 x_{4}$ and $x_{6}$ is TWS, it can be seen from Fig. 7 which based on fine sorting, the threat degree of emitter $x_{3} 、 x_{4}$ and $x_{6}$ is: $x_{6}>x_{4}>$ $x_{3}$.

All the above analysis have justified an obvious view that the result of the algorithm proposed in this paper is consistent with the actual analysis, therefore, the proposed algorithm has preferable reasonability and correctness. In order to further verify the reliability and effectiveness of the algorithm proposed, bring the data information in Table 1 into literature [7], and compute the threat evaluation of emitters $x_{1}-x_{6}$. Finally, obtain the comparison of the two different algorithms, and the result is shown as Fig. 8.

In addition, compute the threat evaluation value ' $\mathrm{K}$ ' of emitters $x_{1}-x_{6}$ in Fig. 8.

$$
\begin{aligned}
& \mathrm{K}\left(x_{1}\right)=1.02, \\
& \mathrm{~K}\left(x_{2}\right)=0.65, \\
& \mathrm{~K}\left(x_{3}\right)=0.92, \\
& \mathrm{~K}\left(x_{4}\right)=0.93, \\
& \mathrm{~K}\left(x_{5}\right)=0.46, \\
& \mathrm{~K}\left(x_{6}\right)=0.94 .
\end{aligned}
$$

According to the threat evaluation value ' $\mathrm{K}$ ', then get the sequence result of emitters $x_{1}-x_{6}: x_{1}>x_{6}>x_{4}>$ $x_{3}>x_{2}>x_{5}$, consequently, the result in literature [7] is consistent with the algorithm proposed in this article. Compare Fig. 7 with Fig. 8, it is obvious that in Fig. 8, the number of index and emitter is more than it in Fig. 7, in the practical calculation, a large number of index and emitter will increase the computation time. In addition, there is some overlap between different radar chart in Fig. 8, therefore, it is not very effortless for us to sort the emitters visually, and the threat evaluation value ' $K$ ' of emitter $x_{3} 、 x_{4}$ and $x_{6}$ is quite close in literature [7],
$\mathrm{K}\left(x_{3}\right)=0.92, \mathrm{~K}\left(x_{4}\right)=0.93, \mathrm{~K}\left(x_{6}\right)=0.94$, which increases the difficulty of ensuring the reliability and effectiveness of threat evaluation. But when analyze the algorithm proposed in this article, fine sorting is based on coarse sorting, and we only need to sort the emitters for which the radar operation mode are same, therefore, the number of index and target emitter in Fig. 7 is less than it in Fig. 8.

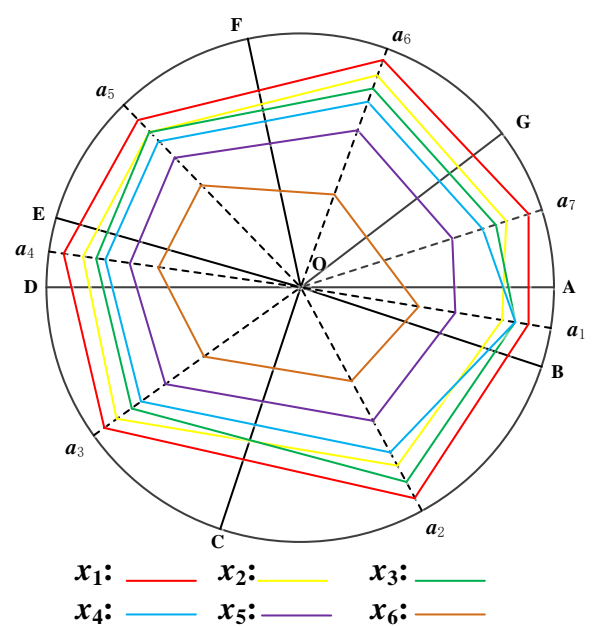

Figure 8. The contrast radar chart of emitters $x_{1}-x_{6}$

In addition, through Fig. 7, it is quite visual and intuitive to sequence the emitters with same radar operation mode. In the meantime, the threat evaluation value ' $\mathrm{K}$ ' of emitter $x_{3} 、 x_{4}$ and $x_{6}$ is: $\mathrm{K}\left(x_{3}\right)=0.79, \mathrm{~K}\left(x_{4}\right)=0.81, \mathrm{~K}\left(x_{6}\right)=0.85$, it is obvious that there is a far cry in the value of ' $K$ ', and the ultimate result of threat evaluation of radiation resource is more reliable and effective than it in literature [7].

\section{Conclusion}

1) A evaluation system of combined threat evaluation of radiation resource is proposed in this article, which combined coarse sorting with fine sorting. Coarse sorting bases on radar operation mode and is regarded as the original threat evaluation, therefore, it's a rough and quick evaluation of threat level. Fine sorting based on improved RCM and focuses on the radiation resources with same radar operation mode. Finally, combine the fine sorting with coarse sorting to obtain a more accurate and reliable result of threat evaluation.

2) A new algorithm based on improved RCM is proposed in this paper, in order to overcome the 
shortage and limitation of traditional radar chart, we determine the size of sector domain of different index according to index's combination weight, meanwhile, IFS is applied to determine the normalized evaluation value of index.

3) Simulation analysis shows the correctness and effectiveness of this algorithm. Compared with classical method of threat evaluation based on RCM, the algorithm proposed in this article is visual in image and can work quickly with lower complexity.

\section{References}

[1] Wang, Y., Liu, S. Y., Zhang, W. et al: Threat Assessment Method with Uncertain Attribute Weight Based on Intuitionistic Fuzzy multiAttribute Decision, ACTA ELECTRONICA SINICA, 42 (2014), 12, 2509-2514.

[2] Meng, G. L., Gong, G. H.: Threat Assessment of Aerial Targets Based on Hybrid Bayesian network, Systems Engineering and Electronics, 32 (2010), 11, 2398-2401.

[3] Wu, H., Su, X. Q.: Threat Assessment of Aerial Targets Based on Group Generalized Intuitionistic Fuzzy Soft Sets, Control and Decision, 30 (2015), 8, 1462-1468.

[4] Chen, J. Y., Yao, P. Y., Wang, B. et al.: Dynamic Threat Assessment Based on Structure Entropy and IGSO-BP Algorithm, Journal of Systems Engineering and Electronics, 37 (2015), 5, 1076-1083.

[5] Wang, H. W., Fan, X. Y., Suo, Z. Y. et al.: A Emitter Threat Evaluating Method Based on Rough Set and TOPSIS, ACTA ARMAMENTARI, 37 (2016), 5, 945-952.

[6] Li, J., Guo, L. H.: Target threat assessment using improved SVM, Optics and Precision
Engineering, 2015, 22(5): 1354-1362.

[7] Zahng, C. K., Zhu, Z. X., Feng, Qi et al. Visualization Threat Assessment for Air Combat Based on Interval-radar Chart, Systems Engineering and Electronic, 38, (2016), 5, 1052-1058.

[8] Li, H.: Improved Combination Weighting radar Chart for Synthetic Evaluation of Power Quality, Journal Of Beijing University Of Technology, 40 (2014), 3, 348-353.

[9] Xhen, Y. X., Cai, Z. Y., Zhang, Z. M. et al. Method for group decision-making information integration based on evidence theory and intuitionistic fuzzy set, Systems Engineering and Electronics, 37 (2015), 3, 594-598.

[10] Zhang, Y., Wang, H. W., Guo, X. T. et al.: A Emitter Threat Assessment Method Based on Intuitionistic Fuzzy Sets and Bayesian Network, Journal of Air Force Engineering University: Natural Science Edition, 18 (2017), 1, 63-68.

[11] Yang, Y. L., Tai, H. X., Shi Tao: Weighting indicators of building energy efficiency assessment taking account of experts' priority, J. Cent. South Univ, 19 (2012), 803-808.

[12] Xu Z.: Theory and Application Research of the Optimization of Equipment Cost and Risk Analysis, Beijing University of Aeronautics and Astronautics, 2006.

[13] Yao, R. P., Shen, H. Z.: Multicriteria Fuzzy Decision Making Method for Interval Valued Intuitionistic Fuzzy Set, Mathematics in practice and theory, 41 (2011), 18, 135-138.

[14] Wang, Y. B., Cheng, S. Y., Zhou, Y. P. et al. Airto-Air Operation Modes Recognition of Airborne Fire Control Radar Based on DS Evidence Theory, Modern radar, 5 (2017), 2: 369-376. 